\title{
Subdirect Sums of Doubly Strictly Diagonally Dominant Matrices
}

\author{
Yating Li, Xiaoyong Chen, Yi Liu, Lei Gao, and Yaqiang Wang $\mathbb{D}$ \\ School of Mathematics and Information Science, Baoji University of Arts and Sciences, Baoji, Shaanxi 721013, China \\ Correspondence should be addressed to Yaqiang Wang; yaqiangwang1004@163.com
}

Received 9 December 2020; Accepted 22 January 2021; Published 8 February 2021

Academic Editor: Ljubisa Kocinac

Copyright (c) 2021 Yating Li et al. This is an open access article distributed under the Creative Commons Attribution License, which permits unrestricted use, distribution, and reproduction in any medium, provided the original work is properly cited.

In this paper, the question of when the subdirect sum of two doubly strictly diagonally dominant (DSDDs) matrices is addressed. Some sufficient conditions are given, and these sufficient conditions only depend on the elements of the given matrices. Moreover, examples are presented to illustrate the corresponding results.

\section{Introduction}

In 1999, the concept of subdirect sums of square matrices was introduced by Fallat and Johnson, which is a generalization of the usual sum of matrices [1], and arises in several contexts, such as matrix completion problems, overlapping subdomains in domain decomposition problems, and global stiffness matrices in finite element methods [2].

For a given class matrix, an important problem is that whether the $k$-subdirect sums of matrices belong to the same class or not, which has been widely concerned for differently classes of matrices, such as nonsingular $M$-matrices [3], $S$-strictly diagonally dominant matrices [4], $\sum$-strictly diagonally dominant matrices [5], doubly diagonally dominant matrices [6], Nekrasov matrices [7, 8], and $\mathrm{SDD}_{1}$ matrices [9].

In this paper, we focus on the subdirect sum of doubly strictly diagonally dominant (shortly as DSDD) matrices, which is a subclass of $H$-matrices [10], and some sufficient conditions such that the $k$-subdirect sums of DSDD matrices belong to DSDD matrices are given, and these sufficient conditions only depend on the elements of the given matrices.

Now, some notations and definitions are listed, which can also be found in $[1,11-13]$.

Let $n$ be an integer number. $C^{n \times n}$ is the set of complex matrices.
Definition 1 (see [1]). Let $A$ and $B$ be square matrices of orders $n_{1}$ and $n_{2}$, respectively, and $k$ be an integer number such that $1 \leq k \leq \min \left\{n_{1}, n_{2}\right\}$. Let $A$ and $B$ be partitioned in a $2 \times 2$ block as follows:

$$
\begin{aligned}
& A=\left[\begin{array}{ll}
A_{11} & A_{12} \\
A_{21} & A_{22}
\end{array}\right], \\
& B=\left[\begin{array}{ll}
B_{11} & B_{12} \\
B_{21} & B_{22}
\end{array}\right],
\end{aligned}
$$

where $A_{22}$ and $B_{11}$ are the square matrices of order $k$. We call the following square matrix of order $n=n_{1}+n_{2}-k$,

$$
C=\left[\begin{array}{ccc}
A_{11} & A_{12} & 0 \\
A_{21} & A_{22}+B_{11} & B_{12} \\
0 & B_{21} & B_{22}
\end{array}\right],
$$

the $k$-subdirect sum of $A$ and $B$, and we denote it by $C=A \oplus_{k} B$.

In order to more explicitly express each element of $C$ in terms of the ones of $A$ and $B$, we can define the following set of indices:

$$
\begin{aligned}
& S_{1}=\left\{1,2, \ldots, n_{1}-k\right\}, \\
& S_{2}=\left\{n_{1}-k+1, n_{1}-k+2, \ldots, n_{1}\right\}, \\
& S_{3}=\left\{n_{1}+1, n_{1}+2, \ldots, n\right\} .
\end{aligned}
$$


Then, $C$ can be expressed as follows:

$$
\begin{aligned}
& C=\left[\begin{array}{ccccccccc}
a_{11} & \cdots & a_{1, t} & a_{1, p} & \cdots & a_{1 n_{1}} & 0 & \cdots & 0 \\
\vdots & \ddots & \vdots & \vdots & \ddots & \vdots & \vdots & \ddots & \vdots \\
a_{t, 1} & \cdots & a_{t, t} & a_{t, p} & \cdots & a_{t, n_{1}} & 0 & \cdots & 0 \\
a_{p, 1} & \cdots & a_{p, t} & a_{p, p}+b_{11} & \cdots & a_{p, n_{1}}+b_{1, n_{1}-t} & b_{1, n_{1}-t+1} & \cdots & b_{1, n-t} \\
\vdots & \ddots & \vdots & \vdots & \ddots & \vdots & \vdots & \ddots & \vdots \\
a_{n_{1} 1} & \cdots & a_{n_{1}, t} & a_{n_{1}, p}+b_{n_{1}-t, 1} & \cdots & a_{n_{1}, n_{1}}+b_{n_{1}-t, n_{1}-t} & b_{n_{1}-t, n_{1}-t+1} & \cdots & b_{n_{1}-t, n-t} \\
0 & \cdots & 0 & b_{n_{1}-t+1,1} & \cdots & b_{n_{1}-t+1, n_{1}-t} & b_{n_{1}-t+1, n_{1}-t+1} & \cdots & b_{n_{1}-t+1, n-t} \\
\vdots & \ddots & \vdots & \vdots & \ddots & \vdots & \vdots & \ddots & \vdots \\
0 & \cdots & 0 & b_{n-t, 1} & \cdots & b_{n-t, n_{1}-t} & b_{n-t, n_{1}-t+1} & \cdots & b_{n-t, n-t}
\end{array}\right], \\
& c_{i j}= \begin{cases}a_{i j}, & i \in S_{1}, j \in S_{1} \cup S_{2}, \\
0, & i \in S_{1}, j \in S_{3}, \\
a_{i j}, & i \in S_{2}, j \in S_{1}, \\
a_{i j}+b_{i-t, j-t}, & i \in S_{2}, j \in S_{2}, \\
b_{i-t, j-t}, & i \in S_{2}, j \in S_{3}, \\
0, & i \in S_{3}, j \in S_{1}, \\
b_{i-t, j-t}, & i \in S_{3}, j \in S_{2} \cup S_{3},\end{cases}
\end{aligned}
$$

where $t=n_{1}-k$ and $p=t+1$.

Definition 2 (see [12]). Given a matrix $A=\left[a_{i j}\right] \in C^{n \times n}, A$ is called (row) diagonally dominant (DD) if

$$
\left|a_{i i}\right| \geq R_{i}(A), \quad i=1,2, \ldots, n,
$$

where

$$
R_{i}(A)=\sum_{\substack{j=1 \\ j \neq i}}^{n}\left|a_{i j}\right|, \quad i=1,2, \ldots, n
$$

If the inequality in (5) holds strictly for all $i$, we say that $A$ is strictly diagonally dominant (SDD).

Definition 3 (see [13]). The matrix $A=\left[a_{i j}\right] \in C^{n \times n}$ is a doubly strictly diagonally dominant (DSDD) matrix if

$$
\left|a_{i i}\right|\left|a_{j j}\right|>R_{i}(A) R_{j}(A), \quad i, j=1,2, \ldots, n, i \neq j .
$$

\section{Subdirect Sums of DSDD Matrices}

In general, the subdirect sum of two DSDD matrices is not always a DSDD matrix. We show this in the following example.
Example 1. Let

$$
\begin{aligned}
& A=\left[\begin{array}{ccc}
1 & 1 & 1 \\
-1 & 4 & 0 \\
-1 & 0 & 4
\end{array}\right], \\
& B=\left[\begin{array}{ccc}
3 & -2 & -1 \\
-1 & 2 & 0 \\
1 & 1 & 4
\end{array}\right]
\end{aligned}
$$

be two DSDD matrices, but

$$
C=A \oplus_{1} B=\left[\begin{array}{cccc}
1 & 1 & 1 & 0 \\
-1 & 7 & -2 & -1 \\
-1 & -1 & 6 & 0 \\
0 & 1 & 1 & 4
\end{array}\right]
$$

is not a DSDD matrix, since $\left|c_{11} \| c_{22}\right|=7<R_{1}(C) R_{2}(C)=8$.

Example 1 shows that the subdirect sum of DSDD matrices is not a DSDD matrix; then, a meaningful discussion is concerned: under what conditions, the subdirect sum of DSDD matrices is in the class of DSDD matrices?

In order to obtain the main results, we need the following lemma. 
Lemma 1. Let $A=\left[a_{i j}\right]$ and $B=\left[b_{i j}\right]$ be DSDD square matrices of orders $n_{1}$ and $n_{2}$ partitioned as in (1), respectively. And, let $k$ be an integer number such that $1 \leq k \leq \min \left\{n_{1}, n_{2}\right\}$, $S_{1}, S_{2}, S_{3}$ be defined as in (4), and all diagonal entries of $A_{22}$ and $B_{11}$ are positive (or all negative), with $C=A \oplus_{k} B$, then,

(a) For $i \in S_{1}$, we have $R_{i}(C)=R_{i}(A)$

(b) Fork $=1, i \in S_{2}$, we have $R_{i}(C)=R_{n_{1}}(C)=$ $R_{n_{1}}(A)+R_{1}(B)$

(c) For $k \geqslant 2, i \in S_{2}$, we have $R_{i}(C) \leqslant R_{i}(A)+R_{i-t}(B)$

(d) For $i \in S_{3}$, we have $R_{i}(C)=R_{i-t}(B)$

Proof. For $i \in S_{1}$, we can write

$$
R_{i}(C)=\sum_{j \in S_{1} j \neq i}\left|a_{i j}\right|+\sum_{j \in S_{2}}\left|a_{i j}\right|+0=R_{i}(A) .
$$

For $k=1, i \in S_{2}=\left\{n_{1}\right\}$, we can obtain

$$
R_{i}(C)=R_{n_{1}}(C)=\sum_{j \in S_{1}}\left|a_{i j}\right|+\sum_{\substack{j \in S_{3} \\ j-t \neq 1}}\left|b_{1, j-t}\right|=R_{n_{1}}(A)+R_{1}(B) .
$$

For $k>2, i \in S_{2}$, we obtain

$$
\begin{aligned}
R_{i}(C)= & \sum_{j \in S_{1}}\left|a_{i j}\right|+\sum_{j \in S_{2} j \neq i}\left|a_{i j}+b_{i-t, j-t}\right|+\sum_{j \in S_{3}}\left|b_{i-t, j-t}\right| \\
\leq & \sum_{j \in S_{1}}\left|a_{i j}\right|+\sum_{j \in S_{2} j \neq i}\left|a_{i j}\right|+\sum_{j \in S_{2} j \neq i}\left|b_{i-t, j-t}\right| \\
& +\sum_{j \in S_{3}}\left|b_{i-t, j-t}\right|=R_{i}(A)+R_{i-t}(B) .
\end{aligned}
$$

For the rest case of $i \in S_{3}$, the proof is similar to the proof of $i \in S_{1}$.

Firstly, we study the 1-subdirect sum of DSDD matrices.

Theorem 1. Let $A$ and $B$ be DSDD matrices of orders $n_{1}$ and $n_{2}$ partitioned as in (1), respectively, and $k=1$. Then, $C=$
$A \oplus_{1} B$ is a DSDD matrix if all diagonal entries of $A_{22}$ and $B_{11}$ are positive (or all negative) and for $i \in S_{1}$,

$$
\begin{gathered}
\left|a_{i i}\right|>\left|a_{n_{1}, n_{1}}\right|, \\
R_{n_{1}}(A) \geq R_{i}(A), \\
\max _{j \in S_{2} \cup S_{3}} \frac{R_{j-t}(B)}{\left|b_{j-t, j-t}\right|} R_{n_{1}}(A) \leq\left|a_{n_{1}, n_{1}}\right|, \quad\left(t=n_{1}-1\right) .
\end{gathered}
$$

Proof. Since $k=1$ and $A$ and $B$ are the DSDD matrices of orders $n_{1}$ and $n_{2}$ respectively, it is obvious that $S_{1}=\left\{1,2, \ldots, n_{1}-1\right\}, S_{2}=\left\{n_{1}\right\}, S_{3}=\left\{n_{1}+1, n_{1}+2, \ldots, n\right\}$.

Case 1: for $i, j \in S_{1}$, from (a) of Lemma 1 , we have

$$
\begin{aligned}
\left|c_{i i}\right| & =\left|a_{i i}\right|,\left|c_{j j}\right|=\left|a_{j j}\right|, \\
R_{i}(C) & =R_{i}(A), \\
R_{j}(C) & =R_{j}(A) .
\end{aligned}
$$

Since $A$ is DSDD, we obtain that for $i, j \in S_{1}$,

$$
\left|c_{i i}\right|\left|c_{j j}\right|=\left|a_{i i}\right|\left|a_{j j}\right|>R_{i}(A) R_{j}(A)=R_{i}(C) R_{j}(C) .
$$

Case 2: for $i \in S_{1}$ and $j \in S_{2}=\left\{n_{1}\right\}$, from (a) of Lemma 1 , it is easy to obtain

$$
\begin{aligned}
\left|c_{i i}\right| & =\left|a_{i i}\right|, \\
R_{i}(C) & =R_{i}(A) .
\end{aligned}
$$

Since all diagonal entries of $A_{22}$ and $B_{11}$ are positive (or all negative), we obtain

$$
\left|c_{j j}\right|=\left|a_{n_{1}, n_{1}}\right|+\left|b_{11}\right| .
$$

Since $A$ is DSDD, and from inequalities (13)-(15), we have

$$
\begin{aligned}
\left|c_{i i i}\right|\left|c_{j j}\right| & =\left|a_{i i}\right|\left(\left|a_{n_{1}, n_{1}}\right|+\left|b_{11}\right|\right)=\left|a_{i i}\right|\left|a_{n_{1}, n_{1}}\right|+\left|a_{i i}\right|\left|b_{11}\right|>R_{i}(A) R_{n_{1}}(A)+\left|a_{n_{1}, n_{1}}\right|\left|b_{11}\right| \\
& \geq R_{i}(A) R_{n_{1}}(A)+\left|b_{11}\right| \max _{j \in S_{2} \cup S_{3}} \frac{R_{j-t}(B)}{\left|b_{j-t, j-t}\right|} R_{n_{1}}(A) \geq R_{i}(A) R_{n_{1}}(A)+R_{n_{1}}(A) R_{1}(B) \\
& \geq R_{i}(A) R_{n_{1}}(A)+R_{i}(A) R_{1}(B)=R_{i}(A)\left(R_{n_{1}}(A)+R_{1}(B)\right) .
\end{aligned}
$$

From (b) of Lemma 1, it is easy to obtain that for $i \in S_{1}, j \in S_{2}=\left\{n_{1}\right\}$,

$$
\left|c_{i i} \| c_{j j}\right|>R_{i}(C) R_{j}(C) .
$$

Case 3: for $i \in S_{1}$ and $j \in S_{3}$, from (a) and (d) of Lemma 1 , we have

$$
\begin{aligned}
\left|c_{i i}\right| & =\left|a_{i i}\right|, \\
R_{i}(C) & =R_{i}(A), \\
\left|c_{j j}\right| & =\left|b_{j-t, j-t}\right|, \\
R_{j}(C) & =R_{j-t}(B) .
\end{aligned}
$$


Then, from inequalities (13)-(15), we have that for $i \in S_{1}$ and $j \in S_{3}$,

$$
\begin{aligned}
\left|c_{i i} \| c_{j j}\right| & =\left|a_{i i}\right|\left|b_{j-t, j-t}\right|>\left|a_{n_{1}, n_{1}}\right|\left|b_{j-t, j-t}\right| \geq\left|b_{j-t, j-t}\right| \max _{j \in S_{2} \cup S_{3}} \frac{R_{j-t}(B)}{\left|b_{j-t, j-t}\right|} R_{n_{1}}(A) \\
& \geq R_{n_{1}}(A) R_{j-t}(B) \geq R_{i}(A) R_{j-t}(B)=R_{i}(C) R_{j}(C) .
\end{aligned}
$$

Case 4: for $i \in S_{2}=\left\{n_{1}\right\}$ and $j \in S_{3}$, from (b) and (d) of Lemma 1 , we obtain

$$
\begin{aligned}
\left|c_{i i}\right| & =\left|a_{n_{1}, n_{1}}\right|+\left|b_{11}\right|, \\
\left|c_{j j}\right| & =\left|b_{j-t, j-t}\right| .
\end{aligned}
$$

Since $B$ is DSDD, and from inequality (15), for $i \in S_{2}=\left\{n_{1}\right\}$ and $j \in S_{3}$, we have

$$
\begin{aligned}
\left|c_{i i}\right|\left|c_{j j}\right| & =\left(\left|a_{n_{1}, n_{1}}\right|+\left|b_{11}\right|\right)\left|b_{j-t, j-t}\right| \\
& =\left|a_{n_{1}, n_{1}}\right|\left|b_{j-t, j-t}\right|+\left|b_{11}\right|\left|b_{j-t, j-t}\right| \\
& >\left|b_{j-t, j-t}\right| \max _{j \in S_{2} \cup S_{3}} \frac{R_{j-t}(B)}{\left|b_{j-t, j-t}\right|} R_{n_{1}}(A)+R_{1}(B) R_{j-t}(B) \\
& \geq R_{n_{1}}(A) R_{j-t}(B)+R_{1}(B) R_{j-t}(B) \\
& =\left(R_{n_{1}}(A)+R_{1}(B)\right) R_{j-t}(B)=R_{i}(C) R_{j}(C) .
\end{aligned}
$$

Case 5: for $i, j \in S_{3}$, from (d) of Lemma 1, we obtain

$$
\begin{aligned}
\left|c_{i i}\right| & =\left|b_{i-t, i-t}\right|, \\
R_{i}(C) & =R_{i-t}(B) .
\end{aligned}
$$

Since $B$ is DSDD, we have

$$
\left|c_{i i}\right|\left|c_{j j}\right|=\left|b_{i-t, i-t}\right|\left|b_{j-t, j-t}\right|>R_{i-t}(B) R_{j-t}(B)=R_{i}(C) R_{j}(C) .
$$

Therefore, we can draw a conclusion that for any $i, j \in\{1,2, \ldots, n\},\left|c_{i i}\right|\left|c_{j j}\right|>R_{i}(C) R_{j}(C)$, that is, $C=A \oplus_{1} B$ is a DSDD matrix.

Example 2. The matrices

$$
\begin{aligned}
& A=\left[\begin{array}{ccc}
3 & 0 & -1 \\
-1 & 4 & 0 \\
-2 & 0 & 2
\end{array}\right], \\
& B=\left[\begin{array}{ccc}
3 & 1 & -1 \\
-1 & -2 & 0 \\
1 & 0 & 2
\end{array}\right]
\end{aligned}
$$

are two DSDD matrices, and from Theorem 1, it is easy to verify that

$$
C=A \oplus_{1} B=\left[\begin{array}{ccccc}
3 & 0 & -1 & 0 & 0 \\
-1 & 4 & 0 & 0 & 0 \\
-2 & 0 & 5 & 1 & -1 \\
0 & 0 & -1 & 2 & 0 \\
0 & 0 & 1 & 0 & 2
\end{array}\right]
$$

is a DSDD matrix since

$$
\begin{aligned}
& \quad 3=\left|a_{11}\right|>\left|a_{33}\right|=2,4=\left|a_{22}\right|>\left|a_{33}\right|=2,2=R_{3}(A)>R_{1} \\
& (A)=1,2=R_{3}(A)>R_{2}(A)=1 \text {, and } \max _{j \in S_{2} \cup S_{3}}\left(R_{j-t}(B) /\right. \\
& \left.\left|b_{j-t, j-t}\right|\right) R_{n_{1}}(A)=\left(R_{3}(B) /\left|b_{33}\right|\right) R_{3}(A)=(4 / 7) \times 3<\left|a_{33}\right| \\
& =2 \text {. } \\
& \quad \text { However, }
\end{aligned}
$$$$
=2 \text {. }
$$

$$
C=A \oplus_{2} B=\left[\begin{array}{cccc}
3 & 0 & -1 & 0 \\
-1 & 7 & 1 & -1 \\
-2 & -1 & 0 & 0 \\
0 & 1 & 0 & 2
\end{array}\right]
$$

is not DSDD since $\left|c_{11} \| c_{33}\right|=0<3=R_{1}(C) R_{3}(C)$.

Examples 2 motivates the search for other conditions such that $C=A \oplus_{k} B(k \geqslant 2)$ is also a DSDD matrix, where $A$ is a DSDD matrix and $B$ is a DSDD matrix. 
Next, some sufficient conditions ensuring that the $k$-subdirect sum of DSDD matrices is a DSDD matrix are given.

Theorem 2. Let $A$ and $B$ be matrices of orders $n_{1}$ and $n_{2}$ partitioned as in (1), respectively, and $k$ is an integer number such that $2 \leq k \leq \min \left\{n_{1}, n_{2}\right\}$. Let $A$ and $B$ be DSDD matrices, if all diagonal entries of $A_{22}$ and $B_{11}$ are positive (or all negative) and for any $i \in S_{1} \cup S_{2}$,

$$
\max _{j \in S_{2} \cup S_{3}} \frac{R_{j-t}(B)}{\left|b_{j-t, j-t}\right|} R_{i}(A)<\left|a_{i i}\right|, \quad\left(t=n_{1}-k\right),
$$

and then the $k$-subdirect sum $C=A \oplus_{k} B$ is DSDD.

Proof. Let $A$ and $B$ be DSDD matrices of orders $n_{1}$ and $n_{2}$ respectively; thus, it is obvious that $S_{1}=\{1,2, \ldots$, $\left.n_{1}-k\right\}, S_{2}=\quad\left\{n_{1}-k+1, n_{1}-k+2, \ldots, n_{1}\right\}, \quad$ and $S_{3}=$ $\left\{n_{1}+1, n_{1}+2, \ldots, n\right\}$.

Case 1: for $i, j \in S_{1}$, from (a) of Lemma 1, we have

$$
\begin{aligned}
\left|c_{i i}\right| & =\left|a_{i i}\right|, \\
R_{i}(C) & =R_{i}(A) .
\end{aligned}
$$

Since $A$ is DSDD, we obtain

$$
\left|c_{i i}\right|\left|c_{j j}\right|=\left|a_{i i}\right|\left|a_{j j}\right|>R_{i}(A) R_{j}(A)=R_{i}(C) R_{j}(C) .
$$

Case 2: for $i \in S_{1}$ and $j \in S_{2}$, from (a) and (c) of Lemma 1 , we obtain

$$
\begin{aligned}
\left|c_{i i}\right| & =\left|a_{i i}\right|, \\
R_{i}(C) & =R_{i}(A), \\
R_{j}(C) & \leq R_{j}(A)+R_{j-t}(B) .
\end{aligned}
$$

Since all diagonal entries of $A_{22}$ and $B_{11}$ are positive (or all negative), we obtain

$$
\left|c_{j j}\right|=\left|a_{j j}+b_{j-t, j-t}\right|=\left|a_{j j}\right|+\left|b_{j-t, j-t}\right| .
$$

Since $A$ is DSDD, and from inequality (31), we have

$$
\begin{aligned}
\left|c_{i i}\right|\left|c_{j j}\right| & =\left|a_{i i}\right|\left(\left|a_{j j}\right|+\left|b_{j-t, j-t}\right|\right)=\left|a_{i i}\right|\left|a_{j j}\right|+\left|a_{i i}\right|\left|b_{j-t, j-t}\right| \\
& >R_{i}(A) R_{j}(A)+\left|b_{j-t, j-t}\right| \max _{j \in S_{2} \cup S_{3}} \frac{R_{j-t}(B)}{\left|b_{j-t, j-t}\right|} R_{i}(A) \\
& \geq R_{i}(A) R_{j}(A)+R_{i}(A) R_{j-t}(B) \\
& =R_{i}(A)\left(R_{j}(A)+R_{j-t}(B)\right) .
\end{aligned}
$$

From (c) of Lemma 1, it is easy to obtain that

$$
\left|c_{i i}\right|\left|c_{j j}\right|>R_{i}(C) R_{j}(C)
$$

Case 3: for $i \in S_{1}$ and $j \in S_{3}$, from (a) and (d) of Lemma 1 , we conclude

$$
\begin{aligned}
\left|c_{i i}\right| & =\left|a_{i i}\right|, \\
R_{i}(C) & =R_{i}(A), \\
\left|c_{j j}\right| & =\left|b_{j-t, j-t}\right|, \\
R_{j}(C) & =R_{j-t}(B) .
\end{aligned}
$$

Then, from inequality (31), we have

$$
\begin{aligned}
& \left|c_{i i}\right|\left|c_{j j}\right|=\left|a_{i i}\right|\left|b_{j-t, j-t}\right| \\
& >\left|b_{j-t, j-t}\right| \max _{j \in S_{2} \cup S_{3}} \frac{R_{j-t}(B)}{\left|b_{j-t, j-t}\right|} R_{i}(A) \\
& \geq R_{i}(A) R_{j-t}(B) \\
& =R_{i}(C) R_{j}(C) .
\end{aligned}
$$

Case 4: for $i, j \in S_{2}$, from (c) of Lemma 1, we obtain

$$
\begin{aligned}
\left|c_{i i}\right| & =\left|a_{i i}\right|+\left|b_{i-t, i-t}\right|, \\
R_{i}(C) & \leq R_{i}(A)+R_{i-t}(B) .
\end{aligned}
$$

Since $A$ and $B$ are DSDD, and from inequality (31), we conclude 


$$
\begin{aligned}
\left|c_{i i}\right|\left|c_{j j}\right| & =\left(\left|a_{i i}\right|+\left|b_{i-t, i-t}\right|\right)\left(\left|a_{j j}\right|+\left|b_{j-t, j-t}\right|\right)=\left|a_{i i}\right|\left|a_{j j}\right|+\left|a_{i i}\right|\left|b_{j-t, j-t}\right|+\left|a_{j j}\right|\left|b_{i-t, i-t}\right|+\left|b_{i-t, i-t}\right|\left|b_{j-t, j-t}\right| \\
& >R_{i}(A) R_{j}(A)+\left|b_{j-t, j-t}\right| \max _{j \in S_{2} \cup S_{3}} \frac{R_{j-t}(B)}{\left|b_{j-t, j-t}\right|} R_{i}(A)+\left|b_{i-t, i-t}\right| \max _{i \in S_{2} \cup S_{3}} \frac{R_{i-t}(B)}{\left|b_{i-t, i-t}\right|} R_{j}(A)+R_{i-t}(B) R_{j-t}(B) \\
& \geq R_{i}(A) R_{j}(A)+R_{i}(A) R_{j-t}(B)+R_{j}(A) R_{i-t}(B)+R_{i-t}(B) R_{j-t}(B) \\
& =\left(R(A)+R_{i-t}(B)\right)\left(R_{j}(A)+R_{j-t}(B)\right) \geq R_{i}(C) R_{j}(C) .
\end{aligned}
$$

Case 5: for $i \in S_{2}$ and $j \in S_{3}$, from (c) and (d) of Lemma 1 , we obtain

$$
\begin{aligned}
\left|c_{i i}\right| & =\left|a_{i i}\right|+\left|b_{i-t, i-t}\right|, \\
R_{i}(C) & \leq R_{i}(A)+R_{i-t}(B), \\
\left|c_{j j}\right| & =\left|b_{j-t, j-t}\right|, \\
R_{j}(C) & =R_{j-t}(B) .
\end{aligned}
$$

Since $B$ is DSDD, and from inequality (31), we obtain

$$
\begin{aligned}
\left|c_{i i}\right|\left|c_{j j}\right| & =\left(\left|a_{i i}\right|+\left|b_{i-t, i-t}\right|\right)\left|b_{j-t, j-t}\right| \\
& =\left|a_{i i} \| b_{j-t, j-t}\right|+\left|b_{i-t, i-t}\right|\left|b_{j-t, j-t}\right| \\
& >\left|b_{j-t, j-t}\right| \max _{j \in S_{2} \cup S_{3}} \frac{R_{j-t}(B)}{\left|b_{j-t, j-t}\right|} R_{i}(A)+R_{i-t}(B) R_{j-t}(B) \\
& \geq R_{i}(A) R_{j-t}(B)+R_{i-t}(B) R_{j-t}(B) \\
& =\left(R_{i}(A)+R_{i-t}(B)\right) R_{j-t}(B) \geq R_{i}(C) R_{j}(C) .
\end{aligned}
$$

Case 6: for $i, j \in S_{3}$, from (d) of Lemma 1, we obtain

$$
\begin{aligned}
\left|c_{i i}\right| & =\left|b_{i-t, i-t}\right|, \\
R_{i}(C) & =R_{i-t}(B) .
\end{aligned}
$$

Since $B$ is DSDD, we obtain

$$
\left|c_{i i}\right|\left|c_{j j}\right|=\left|b_{i-t, i-t}\right|\left|b_{j-t, j-t}\right|>R_{i-t}(B) R_{j-t}(B)=R_{i}(C) R_{j}(C) .
$$

In conclusion, for any $i, j \in S_{1} \cup S_{2} \cup S_{3},\left|c_{i i}\right|\left|c_{j j}\right|>R$ ${ }_{i}(C) R_{j}(C)$. Therefore, $C=A \oplus_{k} B$ is a DSDD matrix.

Example 3. Let

$$
\begin{aligned}
& A=\left[\begin{array}{ccc}
4 & 1 & 4 \\
1 & 5 & -2 \\
0 & -1 & 2
\end{array}\right], \\
& B=\left[\begin{array}{ccc}
2 & 0 & 1 \\
3 & 8 & 0 \\
-1 & 2 & 5
\end{array}\right]
\end{aligned}
$$

be two DSDD matrices. And from Definition 1, we obtain that

$$
C=A \oplus_{2} B=\left[\begin{array}{cccc}
4 & 1 & 4 & 0 \\
1 & 7 & -2 & 1 \\
0 & 2 & 10 & 0 \\
0 & -1 & 2 & 5
\end{array}\right]
$$

and $S_{1}=\{1\}, S_{2}=\{2,3\}$, and $S_{3}=\{4\}$. From Theorem $2, C=$ $A \oplus_{2} B$ is a DSDD matrix since

$$
\max _{j \in S_{2} \cup S_{3}} \frac{R_{j-t}(B)}{\left|b_{j-t, j-t}\right|} R_{1}(A)=\frac{R_{3}(B)}{\left|b_{33}\right|} R_{1}(A)=\frac{3}{5} \times 5<\left|a_{11}\right|,
$$

$$
\max _{j \in S_{2} \cup S_{3}} \frac{R_{j-t}(B)}{\left|b_{j-t, j-t}\right|} R_{2}(A)=\frac{R_{3}(B)}{\left|b_{33}\right|} R_{2}(A)=\frac{3}{5} \times 3<\left|a_{22}\right|
$$

$$
\max _{j \in S_{2} \cup S_{3}} \frac{R_{j-t}(B)}{\left|b_{j-t, j-t}\right|} R_{3}(A)=\frac{R_{3}(B)}{\left|b_{33}\right|} R_{3}(A)=\frac{3}{5} \times 1<\left|a_{33}\right|
$$


From Definitions 2 and 3, it is easy to show that SDD matrices are contained into DSDD matrices. Therefore, from Theorem 2, we obtain the following corollaries, which present sufficient conditions such that $k$-subdirect sum $C=$ $A \oplus_{k} B$ is DSDD.

Corollary 1. Let $A$ and $B$ be square matrices of orders $n_{1}$ and $n_{2}$ partitioned as in (1), respectively, and $k$ is an integer number such that $1 \leq k \leq \min \left\{n_{1}, n_{2}\right\}$. We assume that $A$ is a DSDD matrix and $B$ is a SDD matrix. If there exists an $i_{0} \in S_{1} \cup S_{2}$ such that

$$
\begin{gathered}
\max _{j \in S_{2} \cup S_{3}} \frac{R_{j-t}(B)}{\left|b_{j-t, j-t}\right|} R_{i_{0}}(A)<\left|a_{i_{0}, i_{0}}\right|<R_{i_{0}}(A), \\
\left|a_{i i}\right| \geqslant R_{i}(A), \quad i \in S_{1} \cup S_{2} \backslash\left\{i_{0}\right\} .
\end{gathered}
$$

And, all diagonal entries of $A_{22}$ and $B_{11}$ are positive (or all negative), then the $k$-subdirect sum $C=A \oplus_{k} B$ is a DSDD matrix.

Proof. Without loss of generality, we can assume $i_{0}=1$ such that $\max _{j \in S_{2} \cup S_{3}}\left(R_{j-t}(B) /\left|b_{j-t, j-t}\right|\right) R_{1}(A)<\left|a_{11}\right|<R_{1}(A)$ and $\left|a_{i i}\right| \geq R_{i}(A), \quad i=2,3, \ldots, n_{1}$.

Case 1: for $i, j \in S_{1}$, from (a) of Lemma 1, we have

$$
\begin{aligned}
\left|c_{i i}\right| & =\left|a_{i i}\right|, \\
\left|c_{j j}\right| & =\left|a_{j j}\right|, \\
R_{i}(C) & =R_{i}(A), \\
R_{j}(C) & =R_{j}(A) .
\end{aligned}
$$

Since $A$ is DSDD, we obtain that for $i, j \in S_{1}$,

$$
\left|c_{i i}\right|\left|c_{j j}\right|=\left|a_{i i}\right|\left|a_{j j}\right|>R_{i}(A) R_{j}(A)=R_{i}(C) R_{j}(C) .
$$

Case 2: for $i \in S_{1}$ and $j \in S_{2}$, from (a) of Lemma 1, it is easy to obtain

$$
\begin{aligned}
\left|c_{i i}\right| & =\left|a_{i i}\right|, \\
R_{i}(C) & =R_{i}(A) .
\end{aligned}
$$

Since all diagonal entries of $A_{22}$ and $B_{11}$ are positive (or all negative), we obtain

$$
\left|c_{j j}\right|=\left|a_{j j}\right|+\left|b_{j-t, j-t}\right| .
$$

If $i=1$, since $A$ is DSDD, and from inequality (49), we can obtain

$$
\begin{aligned}
\left|c_{11}\right|\left|c_{j j}\right| & =\left|a_{11}\right|\left(\left|a_{j j}\right|+\left|b_{j-t, j-t}\right|\right)=\left|a_{11}\right|\left|a_{j j}\right|+\left|a_{11}\right|\left|b_{j-t, j-t}\right| \\
& >R_{1}(A) R_{j}(A)+\left|b_{j-t, j-t}\right| \max _{j \in S_{2} \cup S_{3}} \frac{R_{j-t}(B)}{\left|b_{j-t, j-t}\right|} R_{1}(A) \geq R_{1}(A) R_{j}(A)+\left|b_{j-t, j-t}\right| \frac{R_{j-t}(B)}{\left|b_{j-t, j-t}\right|} R_{1}(A) \\
& =R_{1}(A) R_{j}(A)+R_{j-t}(B) R_{1}(A)=R_{1}(A)\left(R_{j}(A)+R_{j-t}(B)\right) \geq R_{1}(C) R_{j}(C) .
\end{aligned}
$$

If $i=2,3, \ldots, n_{1}-k$, since $B$ is $\mathrm{SDD}$, and from inequality (50), we can write

$$
\left|c_{i i}\right|\left|c_{j j}\right|=\left|a_{i i}\right|\left(\left|a_{j j}\right|+\left|b_{j-t, j-t}\right|\right)>R_{i}(A)\left(R_{j}(A)+R_{j-t}(B)\right) \geq R_{i}(C) R_{j}(C) .
$$

Case 3: for $i \in S_{1}$ and $j \in S_{3}$, from (a) and (d) of Lemma 1 , we have

$$
\left|c_{11}\right|\left|c_{j j}\right|=\left|a_{11}\right|\left|b_{j-t, j-t}\right|>\left|b_{j-t, j-t}\right| \max _{j \in S_{2} \cup S_{3}} \frac{R_{j-t}(B)}{\left|b_{j-t, j-t}\right|} R_{1}(A)
$$

$$
\begin{aligned}
\left|c_{i i}\right| & =\left|a_{i i}\right|, \\
R_{i}(C) & =R_{i}(A), \\
\left|c_{j j}\right| & =\left|b_{j-t, j-t}\right|, \\
R_{j}(C) & =R_{j-t}(B) .
\end{aligned}
$$

$$
\begin{aligned}
& \geq\left|b_{j-t, j-t}\right| \frac{R_{j-t}(B)}{\left|b_{j-t, j-t}\right|} R_{1}(A)=R_{1}(A) R_{j-t}(B) \\
& =R_{1}(C) R_{j}(C) .
\end{aligned}
$$

If $i=1$, from inequality (49), we have

If $i=2, \ldots, n_{1}-k$, since $B$ is $\mathrm{SDD}$, and from inequality (50), we can write 


$$
\left|c_{i i}\left\|c_{j j}|=| a_{i i}\right\| b_{j-t, j-t}\right|>R_{i}(A) R_{j-t}(B)=R_{i}(C) R_{j}(C) .
$$

Case 4: for $i, j \in S_{2}$, from (b) and (c) of Lemma 1, we obtain

$$
\begin{aligned}
\left|c_{i i}\right| & =\left|a_{i i}\right|+\left|b_{i-t, i-t}\right|, \\
R_{i}(C) & \leq R_{i}(A)+R_{i-t}(B) .
\end{aligned}
$$

Since $B$ is SDD, and from inequality (50), we can obtain

$$
\begin{aligned}
\left|c_{i i}\right|\left|c_{j j}\right| & =\left(\left|a_{i i}\right|+\left|b_{i-t, i-t}\right|\right)\left(\left|a_{j j}\right|+\left|b_{j-t, j-t}\right|\right) \\
& >\left(R_{i}(A)+R_{i-t}(B)\right)\left(R_{j}(A)+R_{j-t}(B)\right) \\
& \geq R_{i}(C) R_{j}(C) .
\end{aligned}
$$

Case 5: for $i \in S_{2}$ and $j \in S_{3}$, from (c) and (d) of Lemma 1 , we obtain

$$
\begin{aligned}
\left|c_{i i}\right| & =\left|a_{i i}\right|+\left|b_{i-t, i-t}\right|, \\
R_{i}(C) & \leq R_{i}(A)+R_{i-t}(B), \\
\left|c_{j j}\right| & =\left|b_{j-t, j-t}\right|, \\
R_{j}(C) & =R_{j-t}(B) .
\end{aligned}
$$

Since $B$ is SDD, and from inequality (50), we obtain

$$
\left|c_{i i}\right|\left|c_{j j}\right|=\left(\left|a_{i i}\right|+\left|b_{i-t, i-t}\right|\right)\left|b_{j-t, j-t}\right|>\left(R_{i}(A)+R_{i-t}(B)\right) R_{j-t}(B) \geq R_{i}(C) R_{j}(C) .
$$

Case 6: for $i, j \in S_{3}$, from (d) of Lemma 1, we obtain

$$
\begin{aligned}
\left|c_{i i}\right| & =\left|b_{i-t, i-t}\right|, \\
R_{i}(C) & =R_{i-t}(B) .
\end{aligned}
$$

Since $B$ is $\mathrm{SDD}$, we can obtain

$$
\left|c_{i i i}\right|\left|c_{j j}\right|=\left|b_{i-t, i-t} \| b_{j-t, j-t}\right|>R_{i-t}(B) R_{j-t}(B)=R_{i}(C) R_{j}(C) .
$$

Therefore, we can draw a conclusion that for any $i, j \in\{1,2, \ldots, n\},\left|c_{i i} \| c_{j j}\right|>R_{i}(C) R_{j}(C)$; that is, $C=A \oplus_{k} B$ is a DSDD matrix.

Corollary 2. Let $A$ and $B$ be square matrices of orders $n_{1}$ and $n_{2}$ partitioned as in (1), respectively, and $k$ is an integer number such that $1 \leq k \leq \min \left\{n_{1}, n_{2}\right\}$. We assume that $A$ is a $S D D$ matrix and $B$ is a DSDD matrix. If there exists a $j_{0} \in S_{2} \cup S_{3}$ such that

$$
\begin{gathered}
\max _{i \in S_{1} \cup S_{2}} \frac{R_{i}(A)}{\left|a_{i i}\right|} R_{j_{0}-t}(B)<\left|b_{j_{0}-t, j_{0}-t}\right|<R_{j_{0}-t}(B), \\
\left|a_{i i}\right| \geq R_{j-t}(B), \quad j \in S_{2} \cup S_{3} \backslash\left\{j_{0}\right\} .
\end{gathered}
$$

And, all diagonal entries of $A_{22}$ and $B_{11}$ are positive (or all negative), then the $k$-subdirect sum $C=A \oplus_{k} B$ is a DSDD matrix.
Proof. Without loss of generality, we can assume $j_{0}=n$ such that $\max _{i \in S_{1} \cup S_{2}}\left(R_{i}(A) /\left|a_{i i}\right|\right) R_{n-t}(B)<\left|b_{n-t, n-t}\right|<R_{n-t}(B)$ and $\left|b_{j-t, j-t}\right| \geq R_{i-t}(B), j=n_{1}-k+1, n_{1}-k+2, \ldots, n-1$.

Case 1: for $i, j \in S_{1}$, from (a) of Lemma 1, we have

$$
\begin{aligned}
\left|c_{i i}\right| & =\left|a_{i i}\right|, \\
\left|c_{j j}\right| & =\left|a_{j j}\right|, \\
R_{i}(C) & =R_{i}(A), \\
R_{j}(C) & =R_{j}(A) .
\end{aligned}
$$

Since $A$ is SDD, we obtain that for $i, j \in S_{1}$,

$$
\left|c_{i i i}\right|\left|c_{j j}\right|=\left|a_{i i}\right|\left|a_{j j}\right|>R_{i}(A) R_{j}(A)=R_{i}(C) R_{j}(C) .
$$

Case 2: for $i \in S_{1}$ and $j \in S_{2}$, from (a) of Lemma 1, it is easy to obtain

$$
\begin{aligned}
\left|c_{i i}\right| & =\left|a_{i i}\right|, \\
R_{i}(C) & =R_{i}(A) .
\end{aligned}
$$

Since all diagonal entries of $A_{22}$ and $B_{11}$ are positive (or all negative), we obtain

$$
\left|c_{j j}\right|=\left|a_{j j}\right|+\left|b_{j-t, j-t}\right| .
$$

Since $A$ is SDD and from inequality (67), we have 


$$
\left|c_{i i}\right|\left|c_{j j}\right|=\left|a_{i i}\right|\left(\left|a_{j j}\right|+\left|b_{j-t, j-t}\right|\right)>R_{i}(A)\left(R_{j}(A)+R_{j-t}(B)\right) \geq R_{i}(C) R_{j}(C) .
$$

Case 3: for $i \in S_{1}$ and $j \in S_{3}$, from (a) and (d) of Lemma 1 , we have

$$
\begin{gathered}
\left|c_{i i}\right|=\left|a_{i i}\right|, \\
R_{i}(C)=R_{i}(A), \\
\left|c_{j j}\right|=\left|b_{j-t, j-t}\right|, \\
R_{j}(C)=R_{j-t}(B) .
\end{gathered}
$$

If $j=n$, from inequality (66), we have

$$
\begin{aligned}
\left|c_{i i}\right|\left|c_{n n}\right| & =\left|a_{i i}\right|\left|b_{n-t, n-t}\right|>\left|a_{i i}\right| \max _{i \in S_{1} \cup S_{2}} \frac{R_{i}(A)}{\left|a_{i i}\right|} R_{n-t}(B) \\
& \geq\left|a_{i i}\right| \frac{R_{i}(A)}{\left|a_{i i}\right|} R_{n-t}(B) \\
& =R_{i}(A) R_{n-t}(B)=R_{i}(C) R_{n}(C) .
\end{aligned}
$$

If $j=n_{1}-k+1, n_{1}-k+2, \ldots, n-1$, since $A$ is SDD, and from inequality (67), we can write

$\left|c_{i i}\right|\left|c_{j j}\right|=\left|a_{i i} \| b_{j-t, j-t}\right|>R_{i}(A) R_{j-t}(B)=R_{i}(C) R_{j}(C)$.
Case 4: for $i, j \in S_{2}$, from (c) of Lemma 1, we obtain

$$
\begin{aligned}
\left|c_{i i}\right| & =\left|a_{i i}\right|+\left|b_{i-t, i-t}\right|, \\
R_{i}(C) & \leq R_{i}(A)+R_{i-t}(B) .
\end{aligned}
$$

Since $A$ is SDD, and from inequality (67), we can obtain

$\left|c_{i i}\right|\left|c_{j j}\right|=\left(\left|a_{i i}\right|+\left|b_{i-t, i-t}\right|\right)\left(\left|a_{j j}\right|+\left|b_{j-t, j-t}\right|\right)$

$>\left(R_{i}(A)+R_{i-t}(B)\right)\left(R_{j}(A)+R_{j-t}(B)\right) \geq R_{i}(C) R_{j}(C)$.

Case 5: for $i \in S_{2}, j \in S_{3}$, from (c) and (d) of Lemma 1, we obtain

$$
\begin{aligned}
\left|c_{i i}\right| & =\left|a_{i i}\right|+\left|b_{i-t, i-t}\right|, \\
R_{i}(C) & \leq R_{i}(A)+R_{i-t}(B), \\
\left|c_{j j}\right| & =\left|b_{j-t, j-t}\right|, \\
R_{j}(C) & =R_{j-t}(B) .
\end{aligned}
$$

If $j=n$, since $B$ is DSDD, and from inequality (66), we have

$$
\begin{aligned}
\left|c_{i i}\right|\left|c_{n n}\right| & =\left(\left|a_{i i}\right|+\left|b_{i-t, i-t}\right|\right)\left|b_{n-t, n-t}\right| \\
& =\left|a_{i i}\right|\left|b_{n-t, n-t}\right|+\left|b_{i-t, i-t}\right|\left|b_{n-t, n-t}\right| \\
& >\left|a_{i i}\right| \max _{i \in S_{1} \cup S_{2}} \frac{R_{i}(A)}{\left|a_{i i}\right|} R_{n-t}(B)+R_{i-t}(B) R_{n-t}(B) \\
& \geq\left|a_{i i}\right| \frac{R_{i}(A)}{\left|a_{i i}\right|} R_{n-t}(B)+R_{i-t}(B) R_{n-t}(B) \\
& =R_{i}(A) R_{n-t}(B)+R_{i-t}(B) R_{n-t}(B) \\
& =\left(R_{i}(A)+R_{i-t}(B)\right) R_{n-t}(B) \geq R_{i}(C) R_{n}(C) .
\end{aligned}
$$

If $j=n_{1}-k+1, n_{1}-k+2, \ldots, n-1$, since $A$ is SDD,

and from inequality (67), we can write

$$
\left|c_{i i}\right|\left|c_{j j}\right|=\left(\left|a_{i i}\right|+\left|b_{i-t, i-t}\right|\right)\left|b_{j-t, j-t}\right|>\left(R_{i}(A)+R_{i-t}(B)\right) R_{j-t}(B) \geq R_{i}(C) R_{j}(C) .
$$


Case 6: for $i, j \in S_{3}$, from (d) of Lemma 1, we obtain

$$
\begin{aligned}
\left|c_{i i}\right| & =\left|b_{i-t, i-t}\right|, \\
R_{i}(C) & =R_{i-t}(B) .
\end{aligned}
$$

Since $B$ is DSDD, we can obtain

$$
\left|c_{i i}\right|\left|c_{j j}\right|=\left|b_{i-t, i-t}\right|\left|b_{j-t, j-t}\right|>R_{i-t}(B) R_{j-t}(B)=R_{i}(C) R_{j}(C) .
$$

In conclusion, for any $i, j \in S_{1} \cup S_{2} \cup S_{3},\left|c_{i i} \| c_{j j}\right|>$ $R_{i}(C) R_{j}(C)$. Therefore, $C=A \oplus_{k} B$ is a DSDD matrix.

\section{Conclusions}

In this paper, some sufficient conditions such that the subdirect sum of DSDD matrices is in the class of DSDD matrices are given. Moreover, numerical examples are also presented to illustrate the corresponding results.

\section{Data Availability}

No data were used to support this study.

\section{Conflicts of Interest}

The authors declare that they have no conflicts of interest.

\section{Authors' Contributions}

All authors jointly worked on the results, and they read and approved the final manuscript.

\section{Acknowledgments}

This work was partly supported by the National Natural Science Foundations of China (31600299), Natural Science Basic Research Program of Shaanxi, China (2020JM-622), the Scientific Research Program funded by the Shaanxi Provincial Education Department (18JK0044), the Science and Technology Project of Baoji (2017JH2-21 and 2017JH2$24)$, the Key Project of Baoji University of Arts and Sciences (ZK2017021 and ZK16050), and the Postgraduate Innovative Research Project of Baoji University of Arts and Sciences (YJSCX20ZD05).

\section{References}

[1] S. M. Fallat and C. R. Johnson, "Sub-direct sums and positivity classes of matrices," Linear Algebra and Its Applications, vol. 288, pp. 149-173, 1999.

[2] R. Bru, F. Pedroche, and D. B. Szyld, "Additive schwarz iterations for Markov chains," SIAM Journal on Matrix Analysis and Applications, vol. 27, no. 2, pp. 445-458, 2005.

[3] R. Bru, F. Pedroche, and D. B. Szyld, "Subdirect sums of nonsingular $M$-matrices and of their inverse," The Electronic Journal of Linear Algebra, vol. 13, no. 1, pp. 162-174, 2005.
[4] R. Bru, F. Pedroche, and D. B. Szyld, "Subdirect sums of S-strictly diagonally dominant matrices," The Electronic Journal of Linear Algebra, vol. 15, no. 1, pp. 201-209, 2006.

[5] R. Bru, L. Cvetković, V. Kostić, and F. Pedroche, "Sums of $\sum$-strictly diagonally dominant matrices," Linear and Multilinear Algebra, vol. 58, no. 1, pp. 75-78, 2010.

[6] Y. Zhu and T. Z. Huang, "Subdirect Sum of doubly diagonally dominant matrices," The Electronic Journal of Linear Algebra, vol. 16, no. 1, pp. 171-182, 2007.

[7] J. Zhao, D. Liu, and R. Y. Hu, "Subdirect sums of Nekrasov matrices and Nekrasov matrices," Advances Applied Mathematices, vol. 64, no. 2, pp. 1-11, 2015.

[8] L. Gao, H. Huang, and C. Q. Li, "Subdirect sums of Nekrasov matrices," Linear and Multilinear Algebra, vol. 64, no. 2, pp. 208-218, 2016.

[9] X. Chen and Y. Wang, "Subdirect sums of $\mathrm{SDD}_{1}$ matrices," Journal of Mathematics, vol. 2020, Article ID 3810423, 20 pages, 2020.

[10] P.-F. Dai, "A note on diagonal dominance, Schur complements and some classes of $\mathrm{H}$-matrices and P-matrices," Advances in Computational Mathematics, vol. 42, no. 1, pp. 1-4, 2016.

[11] R. A. Horn and C. R. Johnson, Matrix Analysis, Cambridge University Press, Cambridge, MA, USA, 1985.

[12] A. Berman and R. J. Plemmons, Nonnegative Matrices in the Mathematical Sciences, Academic Press, New York, NY, USA, 1979.

[13] B. Li and M. J. Tsatsomeros, "Doubly diagonally dominant matrices," Linear Algebra and Its Applications, vol. 261, no. 1-3, pp. 221-235, 1997. 